\title{
Packet-Layer Forward Error Correction Coding for Fading Mitigation
}

\author{
Hennes Henniger ${ }^{* a}$ \\ ${ }^{a}$ German Aerospace Center (DLR), Institute of Communications and Navigation, \\ Oberpfaffenhofen, 82234 Wessling, Germany
}

\begin{abstract}
In an on/off-keying atmospheric optical communication system, index of refraction turbulence and PAT (pointing, acquisition and tracking) instabilities create strong and very slow fading compared to the usually used high data-rates. With this channel behavior, forward error correction can only work effectively if the codeword-length is longer than the mean duration of fade. Therefore packet-layer coding is a favorable way to implement a code with codewords longer than typical fading events. A code validation platform based on Ethernet transmission technology and packet-layer coding using UDP packets was built up to prove the performance of packet-layer coding. In this paper a demonstrator setup as well as results from a free-space transmission experiment are presented.
\end{abstract}

Keywords: forward error correction, fading mitigation, packet-layer coding

\section{INTRODUCTION}

The work presented in this paper relates to the ATENAA project [1], funded by the European Union, aiming to establish broadband wireless optical communication for commercial aviation.

Free-space optical communication demands robust transmission schemes. As optical wave propagation, especially in the boundary layer, is perturbed by slow fading produced by index of refraction turbulence, transmission requires a means of mitigation. Requirements for mobile terminals impede the use of large receiver apertures or spatial-diversity techniques. Therefore channel coding arises as a suitable mitigation technique.

For typically used high data-rates well above $100 \mathrm{Mbps}$ and typical channel coherence times of some milliseconds, physical-layer bit-wise coding can not deal with burst errors longer than codewords [2]. Further, interleaver with practically feasible depth operating in the physical-layer fail in splitting long burst errors produced by slow fading. This problem can be overcome by an alternative approach, where packet-layer (OSI: transport-layer) issues are considered. Using packet-layer coding instead of bits, packets (transport-layer-symbols) are seen as code-symbols. It is reasonable to propose a channel coding at the packet-layer with implicitly longer symbol-transmission times of the same order as channel coherence time. Typical time constants for fading, physical- and packet-layer coding codewords are compared in figure 1.

Packet-layer coding works in principle the same way as physical-layer coding: codewords consisting of symbols. The difference is that for physical-layer coding, symbols are typically bits whereas for packet-layer coding whole packets are seen as symbols. Considering for example an Ethernet packet with a length of 1000 byte as a symbol, the transmission time of a symbol is $8 \mu$ s assuming a 1 bps channel data rate, and $0.8 \mathrm{~ms}$ for $10 \mathrm{Mbps}$. So these time constants, especially for lower data-rates, are on the same order of magnitude as atmospheric turbulence fluctuations or tracking instabilities

\footnotetext{
* hennes.henniger@dlr.de, phone +49 815328 1147; fax +49 815328 2844; www.dlr.de/kn/

Copyright 2006 Society of Photo-Optical Instrumentation Engineers. This paper was published in Proceedings of the SPIE Vol. 6304 and is made available as an electronic reprint with permission of SPIE. One print or electronic copy may be made for personal use only. Systematic or multiple reproduction, distribution to multiple locations via electronic or other means, duplication of any material in this paper for a fee or for commercial purposes, or modification of the content of the paper are prohibited.
} 
(channel coherence time: 0.5 to $10 \mathrm{~ms}$ ). For detailed description of packet-layer coding for free-space optics please refer to [3].

For simplex optical links using Ethernet transmission technology is an easy way to implement a packet orientated transmission system. Ethernet provides asynchronous transmission with data-rates of $10 \mathrm{Mbps,} 100 \mathrm{Mbps}$ and 1 Gbps fitting quite well to experimental FSO systems. Based on Ethernet, an IP/UDP transmission system can be set up. For UDP-packet transmission open source software libraries exist [4] which allow easily programmed encoder and decoder algorithms for packet-layer coding. The problem is that even UDP based on IP and Ethernet is not really a simplex transmission scheme because Ethernet is using bidirectional link test procedures before sending packets at physical layer.

The purpose of this paper is to present the setup of an Ethernet based packet-layer coding performance simulator and free-space optical data transmission demonstrator. Details of a conversion-device enabling simplex Ethernet transmission over a free-space optical link are given. In the last section, results of the transmission tests are compared with simulation results.

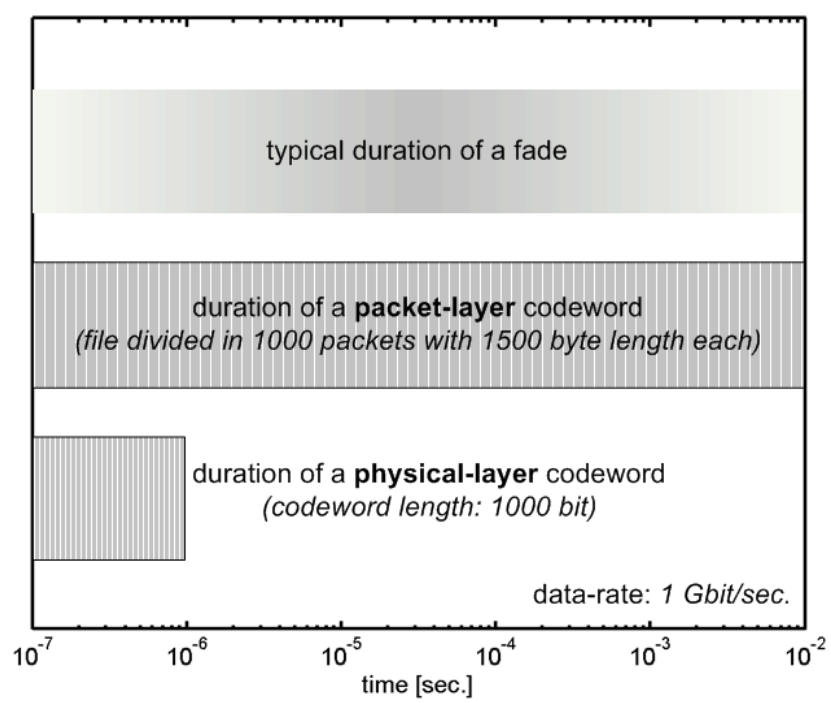

Figure 1: Comparison of typical fade duration (respectively channel coherence time) with packet-layer codeword length and physical-layer codeword-length.

\section{ETHERNET / SIMPLEX DATA-STREAM MEDIA-CONVERSION}

For the purpose of transmitting Ethernet packets over a simplex free-space optical on/off-keying (OOK) link, special media-conversion devices (MCD) were developed using commercial off-the-shelf (COTS) Ethernet electro-optical media-converters usually used in terrestrial fiber systems.

For the transmission of Ethernet packets over the electrical network interface of a personal computer, the standards 10BASE-TX or 100BASE-TX are applied. Both types use a shielded twisted-pair wire for data transmission. The media-converters can automatically determine the ability of the connected computer and choose the correct link speed by auto-negotiation. The standard used for transmission over optical fiber connections is called 100BASE-FX. 100BASE-FX is a version of Fast Ethernet over optical fiber. It uses two separated physical channels for receive and transmit, e.g. two fibers or two wavelengths. For signal coding, two methods are applied transmitting Ethernet packets over an optical fiber link: 4B5B for line coding and NRZ-I (non return to zero inverse) for symbol coding. 4B5B coding is used to avoid long sequences of ones or zeros. Through this there is a data-rate of $125 \mathrm{Mbps}$ on the optical channel while the user data-rate is still 100 Mbps. NRZ-I is a method to transmit a bit pattern over a transmission line. By 
transmitting a logical zero, the status of the line is left unchanged. If a logical one is transmitted, the level on the transmission line is changed. The usage of the combination of 4B5B and NRZ-I ensures that the signal level changes every two bits. If no data is present to transmit over the optical link, the COTS media-converters send a square-wave link-setup-signal with a data rate of $125 \mathrm{Mbps}$. (Therefore continuous signal transmission of the laser-terminal is ensured an a beaconless pointing, acquisition and tracking concept can be applied.) This signal contains a code violation, which results in every 84th bit being longer. According to the data-rate, these bits occurs approximately every 700 ns. If a COTS media-converter receives a square-wave link-setup-signal with extended bits from the link partner, it switches over to a continuous square-wave signal transmission without extended bits. This idle-signal is sent continuously if no data is transmitted and is used for clock synchronization on the receiver side. Receiving the idlesignal, the receiver knows that the link is not broken and the media-converter is also ready to receive data packets. It can be seen that COTS media-converters are using a bidirectional link test procedure by using link-setup-signal and idlesignal. Therefore no Ethernet data packets can be sent if no link-setup signal is detected from the partner. This means that on the physical-layer, a bidirectional channel is needed to connect two COTS media-converters. Hence, optical Ethernet (100BASE-FX) is basically not working on a real simplex channel. As a COTS media-converter is always ready to receive, the link-setup signal or data packets, using a simplex channel is only a problem on the transmitter-side, because no packets will be send to a broken link. This problem can be solved using two receiver-media-converters (see LCS-C842TS-20 devices in figure 2): One local receiver-media-converter at the transmitter-side which generates the feedback, feigns an available link to the transmitter-media-converter (LCS-C842RS-20) locally; and one receivermedia-converter on the receiver-side of the free space optical link. If data is transmitted, it is multiplexed into the idlesignal. The synchronisation preamble before each packet, which is know from 100-BASE TX, is not required and thus is omitted, because a signal for clock synchronization is already present. A transmitted Ethernet packet starts with a 1 byte start-delimiter which in coded form consists of four zeros and four ones. After that the usual data blocks used in an Ethernet-frame are transmitted. The types of transmitted blocks are similar to the ones used in the FDDI-Standard.

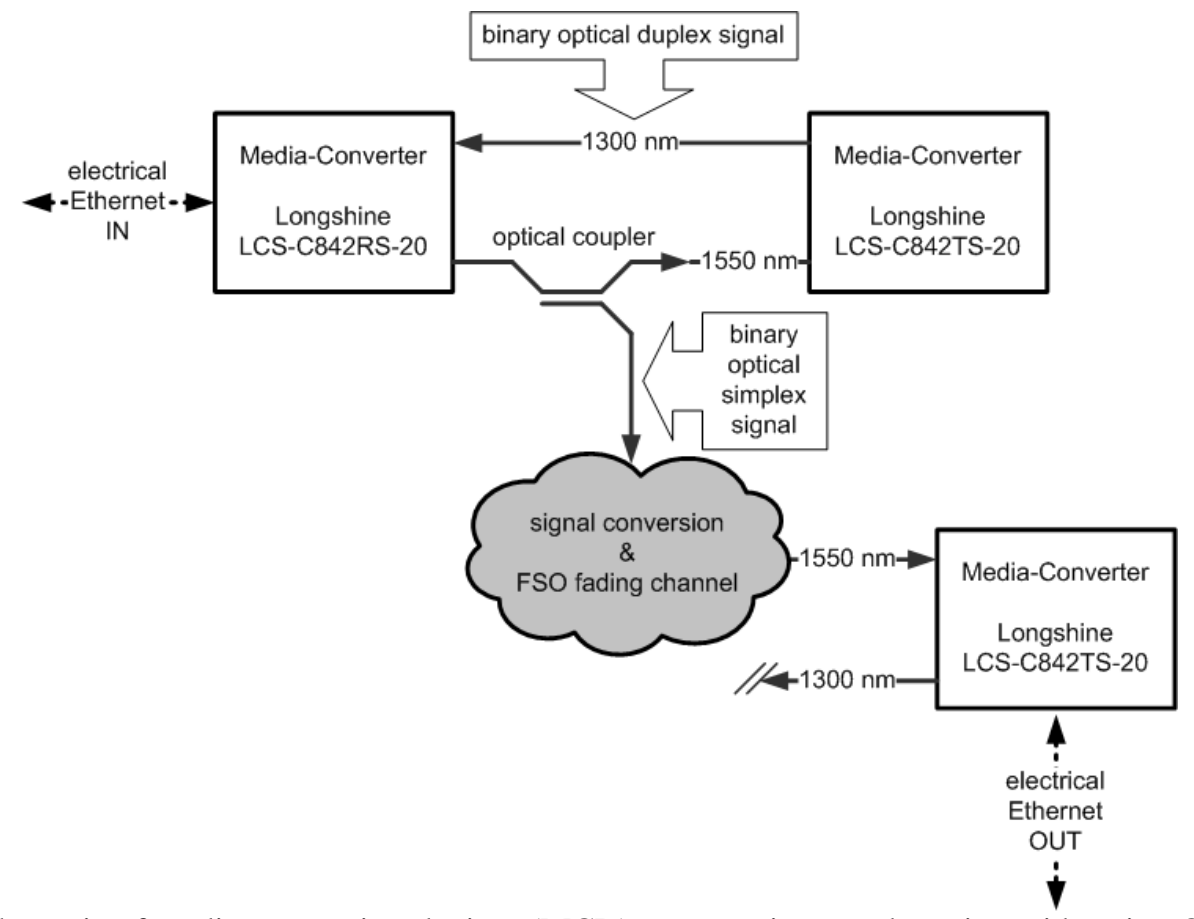

Figure 2: Schematic of media-conversion-devices (MCD) at transmitter- and receiver-side using COTS mediaconverters. 


\section{SETUP OF THE FREE-SPACE OPTICAL TRANSMISSION EXPERIMENT}

In this chapter the experimental setup is described. An overview of the system used is given in Figure 3. Files are spit and packed into UDP packets. UDP packets are coded using standard personal-computers (Intel P4, 3.4 GHz; 2048 MByte RAM) and transmitted based on Ethernet packets. The Ethernet packet stream is converted by a mediaconversion device (MCD) in a real simplex asynchronous serial binary data stream. A binary NRZ signal is transmitted over an intensity-modulated free-space optical link.

The service application implemented here for demonstration and validation continuously mirrors all files (size some kilobyte to 150 Mbyte) stored in a certain folder at the transmitter-side to the receiver-side. In an aircraft application this can be used for example to download all videos available at a high-altitude-platform (HAP) to an aircraft, where the videos are cached at a server for further onboard video streaming.

The horizontal test link is located at the DLR premises Oberpfaffenhofen, near Munich at an altitude of about $600 \mathrm{~m}$ and height of about $15 \mathrm{~m}$ above ground. The link is a double-path link using a retro-reflector $508 \mathrm{~m}$ away from the transmitter. The link passed over asphalt, grass and buildings. The weather conditions on the test day were overcast, rainy and windy.

The transmit assembly (TXA) consists of a $808 \mathrm{~nm}$ multimode fiber-coupled, direct modulated semiconductor laser diode. Transmit power is adjusted with a maximum value of $1 W_{C W}$ to get different transmission quality. The optical beam is collimated and the FWHM divergence angle is measured to be 2.4 mrad.

The receive assembly (RXA) consists of a $20 \mathrm{~mm}$ aperture diameter lens, focusing the incoming light to an APDfrontend. Background-light is blocked by a $750 \mathrm{~nm}$ optical long-pass filter. The electrical signal is filtered by an $80 \mathrm{MHz}$ low-pass filter and a Limiting-Amplifier is used for signal conditioning.

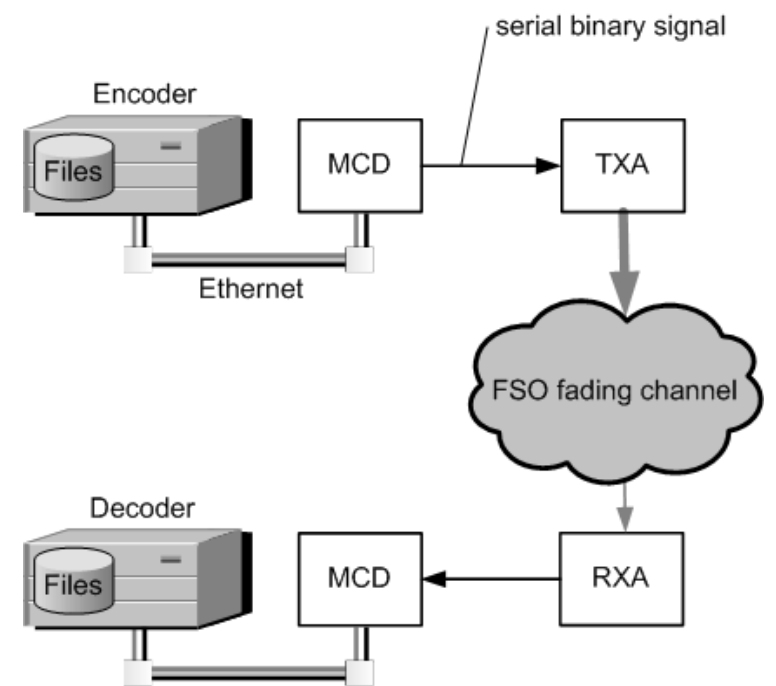

Figure 3: Setup of the free-space transmission experiment.

Ethernet uses a simple CRC (cyclic redundancy check) and therefore only error-free packets are handed on by the MCD. Whether a single bit-error or a bit-error-burst occurs within a packet the packet is discarded. Hence channel performance is measured in terms of packet erasure probability $p_{e}$ which is the ratio lost to transmitted packets. During the measurements the best packet erasure values are in the order of $10 \%$ to $30 \%$. However, the channel was made worse by decreasing transmit power. 


\section{SETUP OF THE CODING SIMULATOR}

The aim of the simulator is to evaluate the performance of LDPC (Low-Density Parity-Check) codes in a channel whose behaviour is similar to turbulent FSO channel. The FSO channel is modelled using a packet erasure channel (PEC). A simplex 10/100/1000BaseT Ethernet link between two computers is built, where $C++$ software emulates the encoding, packet erasure at transmission and decoding of data files through a point to point channel. For the encoder and decoder software the LDPC library contained in the Open-Source MCLv3 package [4] is used. In order to achieve the most reliable results, the Ethernet link between the two computers is forced to be simplex. The Auto-Negotiation protocol is switched off and the transmission rate could be fixed to the Ethernet rates of $10 \mathrm{Mbps}, 100 \mathrm{Mbps}$ or $1000 \mathrm{Mbps}$. To really ensure a simplex communication, the return link inside the RJ45-Crossover Ethernet cable is electrically not connected, and the Normal Link Pulses (NLP) needed by the encoder-PC (PC1) Network Interface Card (NIC) to test the channel's availability is produced by a second NIC placed in the same PC. The demonstrator setup is shown in figure 4. The ARP (Address Resolution Protocol) cache of the encoder-PC (PC1) must contain the NIC physical address (MAC) of the decoding computer (PC2). Thus, one avoids blocking the encoding computer (PC1) in a standby state waiting for the ARP datagram containing the mentioned MAC address of the decoder (PC2). As far as simulations are concerned, the FSO packet erasure channel is represented by deleting a certain amount of packets in PC2 before the decoding process starts. This is done by a randomly generated binary erasure vector. A " 1 ” in the vector means that a packet is discarded and " 0 " means that a packet is transmitted error-free. The probability of erasure is equal to the number of " 1 " in the vector divided by the vector length. The fade length can be chosen by generating series of " 1 ”. Fade duration is measured in packet-lengths.

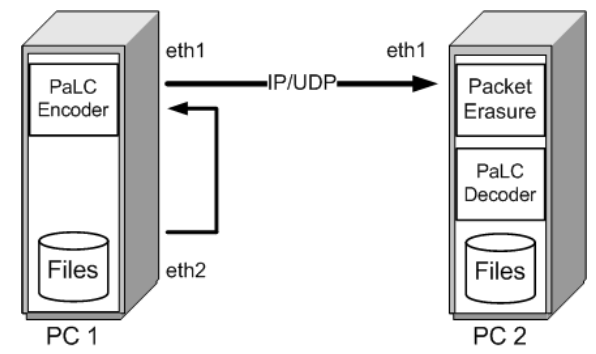

Figure 4: Setup of the Ethernet-based coding simulator

\section{COMPARISON OF MEASUREMENT AND SIMULATION RESULTS}

Coding performance is measured in terms of packet-error-ratio (PER). The PER is defined as the ratio of the number of data packets not recovered after decoding to the number of data packets transmitted. A parity packet that is lost and not recovered is neglected in the PER. In the literature PER is often also called SER (symbol-error-ratio). SER can be misunderstood because a symbol can either be a bit or a packet.

A LDGM staircase code with degree 3 and a packet length of 400 byte or 1500 byte is transmitted over the free-space test channel described above. For different packet erasures, the performance of the code is tested. Figure 5 (left) displays codes which achieve a zero packet-error-erasure after decoding for different channel packet erasures. It can be seen that the following rule of thumb is true: For error-free transmission of data it can be said that the code needs a redundancy which is about $10 \%$ more than the expected probability of erasure. If the code has more than the required redundancy it also works well, but the coding overhead should be reduced as much as possible in order to enhance the channel throughput. The optimum case is approximately given by the rule stated above.

Figure 5 (right) shows packet erasure rates (PER) of a code for different probability of packet erasure. It is clearly shown that using a code with a $50 \%$ redundancy, all files transmitted correctly, under channel conditions of $40 \%$ packet erasure. Therefore again the given rule of thumb is demonstrated to be true.

In figure 6 codes with different degrees and different code-types are compared. The test results are close-by the simulated results. The LDGM staircase code with degree 3 seems to perform best in the free-space optical channel. 
Encoding and decoding times were also measured by the simulator. Results show that LDGM triangle has an encoding/decoding delay approximately double the LDGM staircase. Further, decoding is always slower than encoding. For a $50 \%$ redundancy LDGM staircase code, the encoding time is $150 \mathrm{~ms}$ (packet-length: 400 byte) and $90 \mathrm{~ms}$ (1500 byte). Decoding time is $280 \mathrm{~ms}$ (400 byte) and $150 \mathrm{~ms}$ (1500 byte). The processing times for LDGM are shorter but LDGM performs worse for low packet-erasures - compare figure 6 (left).

The family of LDPC codes available in MCLv3 [4] was evaluated in terms of code efficiency by means of simulations. A file with 13.7 Mbyte was transmitted over a channel with $40 \%$ packet erasure. The packet-length was fixed to be 1500 byte. Figure 7 shows that in general LDGM staircase outperform LDGM triangle and LDGM staircase. For low redundancy values (20\% to $45 \%$ ), LDGM seems to be favourable because it achieves lower PER and processing time is low. In contrast LDGM staircase and LDGM triangle achive error-free transmission for redundancy values larger than approximately $45 \%$ (packet erasure plus $5 \%$ ). The advantage of LDGM contrasts with the behaviour that error-free transmission is not reached for high redundancy values. Therefore LDGM is good for improving the PER when using low redundancy, but it is not suitable for achieving error-free transmission. Therefore the other two types of codes are preferable.
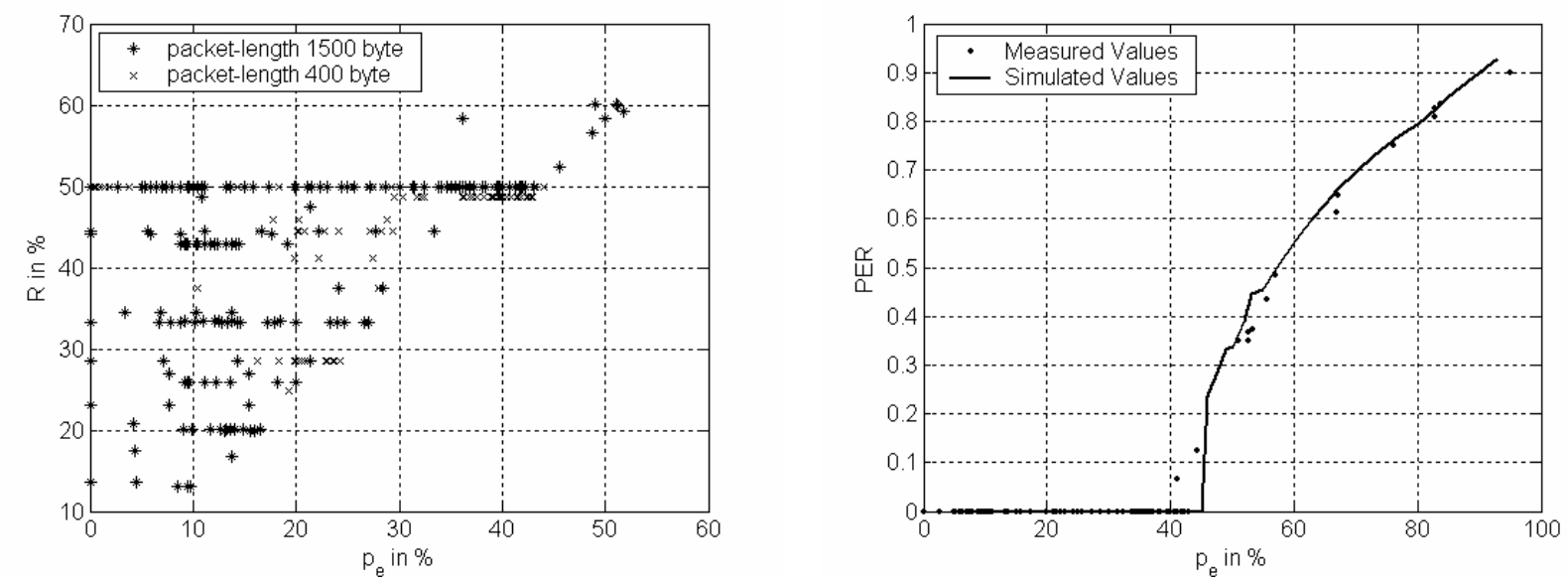

Figure 5: Packet-layer-coding performance: Redundancy of codes which perform error free depending on the channel packet erasure (left); packet-error-ratio vs. probability of packet erasure for a code with $50 \%$ redundancy and a packet-length of 1500 byte (right); (Code: LDGM staircase, degree 3, block-interleaving over whole file)
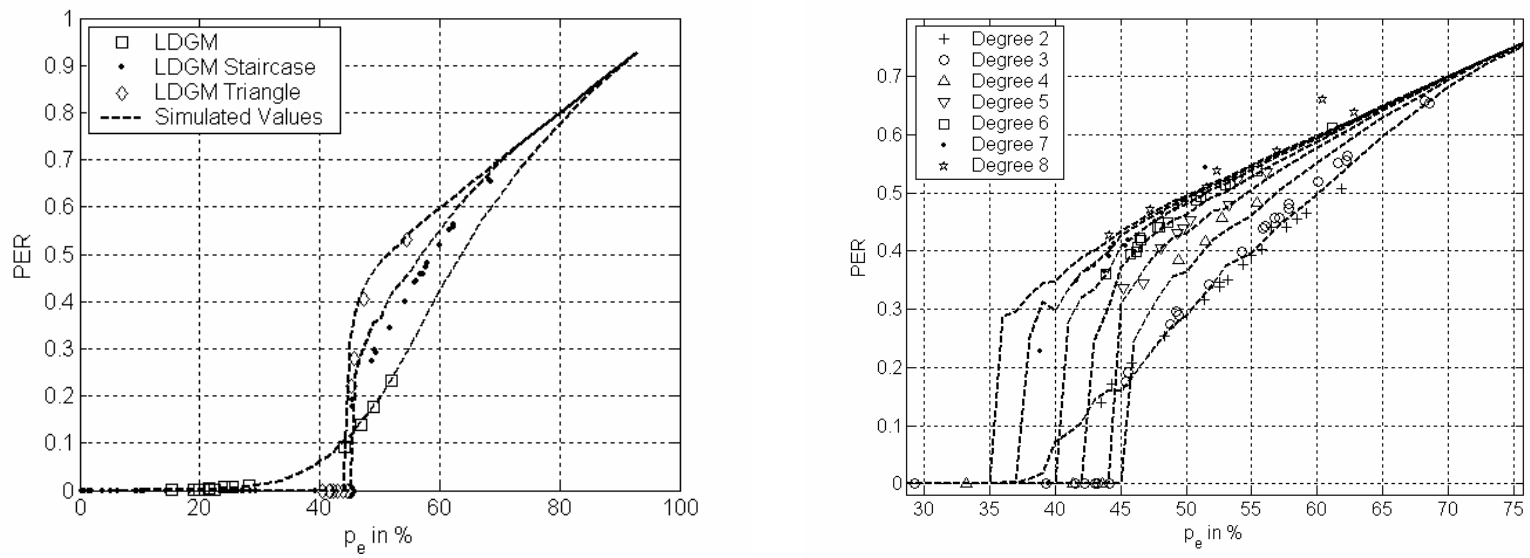

Figure 6: Packet-error ratio vs. probability of packet erasure for various packet erasures and different code-types (left); comparison of the performance of LDGM staricase codes with different degrees (right); (packet-length 400 bytes, degree 3 , redundancy $50 \%$, block-interleaving over whole file). 


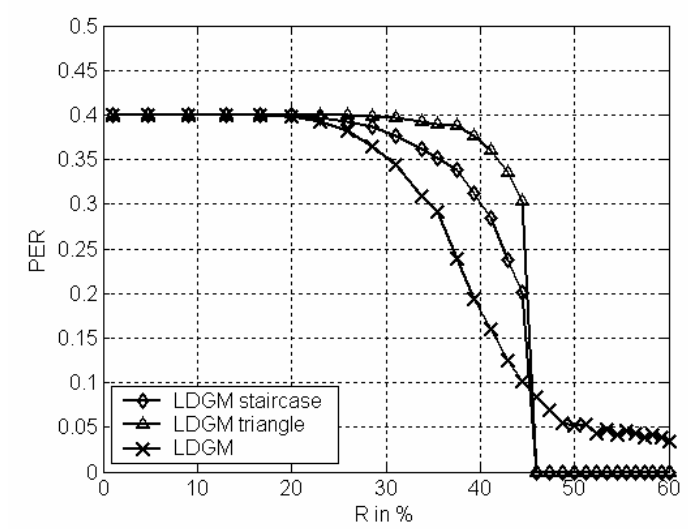

Figure 7: Comparison of LDGM, LDGM staircase and LDGM triangle in terms of code efficiency (degree 3, packet-erasure $40 \%$, block-interleaving over whole file)

When several consecutive packets are affected by a fade, interleaving can be applied to scramble the packet errors over the whole codeword. This will ease the decoding process. In the following simulation, fades with a length equal to 33 times the packet length are simulated by discarding consecutive packets before decoding. In the interleaved case, all packets of a file are scrambled by a block-interleaver. In figure 8, an interleaver gain can be seen. Approximately $8 \%$ less redundancy is needed for error-free transmission.

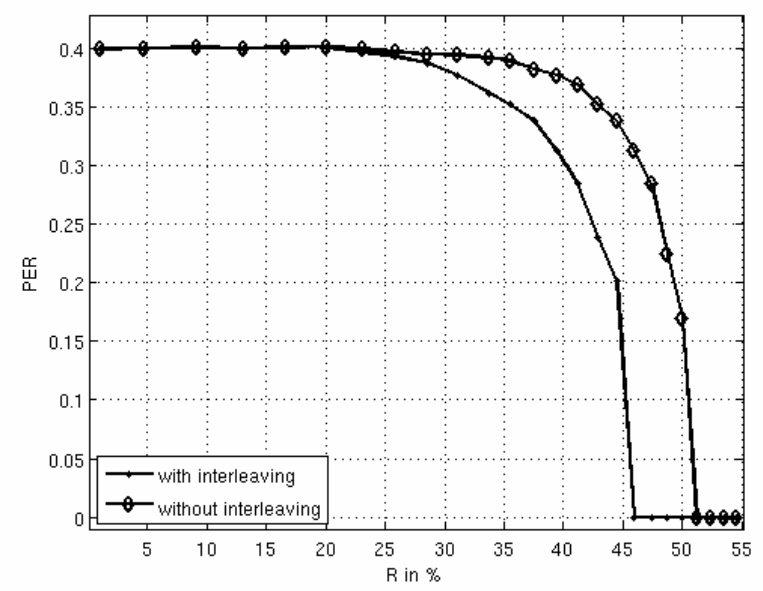

Figure 8: Interleaving gain: Packet-error-rate vs. redundancy for a file transmission with LDGM staircase (degree 3, packet-erasure $40 \%$, packet-length 1500 byte, block-interleaving over whole file).

Measurements and simulations in this section show that channel coding using packet-layer codes can assure error-free transmission in an atmospheric optical fading channel even using high data-rates. The results described agree with theoretical investigations and experiments carried out in [5].

\section{CONCLUSION}

In this paper, simulation results for packet-layer coding are validated with results from a real transmission experiment with good compliance. Packet-layer coding is a promising fading mitigation technique for the free-space optical fading channel where the code has to deal with long channel coherence times. Most investigations were done for high code 
redundancies, but the results can also be applied for codes with lower redundancy if the channel packet-erasure is lower. Ethernet transmission technology is a good way to implement packet-layer coding for free-space optical systems using off-the-shelf-components. Further investigations and tests will be done in future in order to develop, for example, an UDP-transparent system and to prove the benefits of packet-layer coding on long distance links.

\section{ACKNOWLEDGMENT}

I wish to thank Albert Gonzalez (Technical University of Catalonia) and Martin Brechtelsbauer (DLR) for their valuable contribution and assistance, especially preparing simulations and measurements.

\section{REFERENCES}

[1] European Community $6^{\text {th }}$ Framework Program: ATENAA, Advanced Technologies for Networking in Avionic Applications. CEC contract number AST3-CT-2004-502843, www.atenaa.org.

[2] H. Henniger, F. David, D. Giggenbach, and C. Rapp: Evaluation of FEC for the Atmospheric Optical IM/DD Channel. Proc. of SPIE, Vol. 4975, Free-Space Laser Communications Technologies XV, 2003.

[3] H. Henniger, A. Gonzalez: Transmission Scheme and Error Protection for Simplex Long-Distance Atmospheric FSO Systems. Special issue of the Mediterranean Journal of Electronics and Communications on Hybrid RF and Optical Wireless Communications, 2006.

[4] C. Neumann, V. Roca, J. Labouré, and Z. Khallouf: MCLv3 an open source gnu/gpl Implementation of LowDensity Parity Check (LDPC) Large Block FEC Code. URL:

http://www.inrialpes.fr/planete/people/roca/mcl/mcl.html, May 2006.

[5] V. Roca, C. Neumann: Design Evaluation and Comparison of Four Large Block FEC Codes, LDPC, LDGM Staircase and LDGM Triangle, plus a Reed-Solomon Small Block FEC Code. Repport de recherche , $\mathrm{n}^{\circ} 5225$, June 2004. 